\title{
BMJ Open Feasibility study to assess the impact of a lifestyle intervention ('LivingWELL') in people having an assessment of their family history of colorectal or breast cancer
}

\author{
Annie S Anderson, ${ }^{1}$ Jacqueline Dunlop, ${ }^{2}$ Stephanie Gallant, ${ }^{1}$ Maureen Macleod, ${ }^{1}$ \\ Zosia Miedzybrodzka, ${ }^{3}$ Nanette Mutrie, ${ }^{4}$ Ronan E O'Carroll, ${ }^{5}$ Martine Stead, ${ }^{6}$ \\ Robert J C Steele, ${ }^{1}$ Rod S Taylor, ${ }^{7}$ Sarah Vinnicombe, ${ }^{1}$ Jonathan Berg ${ }^{8}$
}

To cite: Anderson AS, Dunlop J, Gallant S, et al. Feasibility study to assess the impact of a lifestyle intervention ('LivingWELL') in people having an assessment of their family history of colorectal or breast cancer. BMJ Open 2018;8:e019410. doi:10.1136/ bmjopen-2017-019410

- Prepublication history for this paper is available online. To view these files, please visit the journal online (http://dx.doi. org/10.1136/bmjopen-2017019410).

Received 31 August 2017 Revised 14 November 2017 Accepted 7 December 2017

Check for updates

For numbered affiliations see end of article.

Correspondence to

Professor Annie S Anderson; a.s.anderson@dundee.ac.uk

\section{ABSTRACT}

Objectives To assess the feasibility of delivering and evaluating a weight management (WM) programme for overweight patients with a family history $(\mathrm{FH})$ of breast cancer (BC) or colorectal cancer (CRC).

Study design A two-arm (intervention vs usual care) randomised controlled trial.

Setting National Health Service (NHS) Tayside and NHS Grampian.

Participants People with a FH of BC or CRC aged $\geq 18$ years and body mass index of $\geq 25 \mathrm{~kg} / \mathrm{m}^{2}$ referred to NHS genetic services.

Intervention Participants were randomised to a control (lifestyle booklet) or 12-week intervention arm where they were given one face-to-face counselling session, four telephone consultations and web-based support. A goal of $5 \%$ reduction in body weight was set, and a personalised diet and physical activity (PA) programme was provided. Behavioural change techniques (motivational interviewing, action and coping plans and implementation intentions) were used.

Primary outcome Feasibility measures: recruitment, programme implementation, fidelity measures, achieved measurements and retention, participant satisfaction assessed by questionnaire and qualitative interviews. Secondary outcomes Measured changes in weight and PA and reported diet and psychosocial measures between baseline and 12-week follow-up.

Results $0 f 480$ patients approached, 196 (41\%) expressed interest in the study, and of those, $78(40 \%)$ patients were randomised. Implementation of the programme was challenging within the time allotted and fidelity to the intervention modest (62\%). Qualitative findings indicated the programme was well received. Questionnaires and anthropometric data were completed by $>98 \%$. Accelerometer data were attained by $84 \%$ and $54 \%$ at baseline and follow-up, respectively. Retention at 12 weeks was $76 \%$. Overall, $36 \%$ of the intervention group (vs $0 \%$ in control) achieved $5 \%$ weight loss. Favourable increases in PA and reduction in dietary fat were also reported.
Strengths and limitations of this study

- This feasibility study is the first attempt to offer and assess a structured, comprehensive lifestyle programme (diet, alcohol, physical activity and body weight) for people referred to family history clinics for colorectal and breast cancer risk assessment.

- The study design is a randomised, two-centred, lifestyle intervention study with subjective and objective assessment measures.

- Participants were all attendees at the National Health Service Family History clinics due to a family history of breast cancer (BC) or colorectal cancer (CRC) and are not representative of the general population.

- The lifestyle intervention was not fully implemented, recruitment was lower than anticipated and indicative findings suggest favourable effects of the intervention on physiological measures.

- The primary and secondary outcome data provide sufficient information to inform a definitive trial.

Conclusions A lifestyle programme for people with a family history of cancer is feasible to conduct and acceptable to participants, and indicative results suggest favourable outcomes.

Trial registration number ISRCTN13123470; Pre-results.

\section{INTRODUCTION}

It is recognised that cancer arises from an interaction between genetic and environmental factors (nature and nurture), although there may be more emphasis given to genetics and family history in the National Health Service (NHS) rather than health behaviour profiles. Clearly, it is desirable that people who are at greater risk of cancer due to a family history of the disease (which may reflect shared genetic and behavioural profiles) are supported to follow recommendations for 
cancer surveillance and lifestyle. NHS genetics centres in Scotland offer early detection and counselling for people with a family history (FH) of breast cancer (BC) and colorectal cancer (CRC), ${ }^{1}$ but there is little evidence of lifestyle advice.

Current estimates for the role of lifestyle in postmenopausal BC suggest that around $38 \%$ of the disease could be prevented by increased physical activity and decreases in alcohol intake and excess body weight. ${ }^{2} \mathrm{~A}$ number of studies show that BC risk is lowered with intentional weight $\operatorname{loss}^{34}$ and recent data from bariatric surgery ${ }^{5}$ show that weight loss is correlated with significant decreases in the incidence of cancers at several sites-notably postmenopausal breast, colon, endometrium and pancreas. Gramling et al reported from the Women's Health Initiative Observational study that participating in healthy lifestyles (greater physical activity, lower alcohol intake and lower body mass index (BMI)) led to risk reduction in postmenopausal women, and the degree of this benefit was the same for women with and without an FH of BC. A recent review by Pettapiece-Phillips $e t a l^{7}$ reports evidence of a protective role of a healthy body size and regular activity among mutation carriers notably in adolescence and early adulthood.

It is estimated that around $45 \%$ of CRC could be prevented by diet and other lifestyle behaviours (dietary fibre intake and physical activity and low intakes of red and processed meat, alcohol and body fatness). ${ }^{2}$ For CRC, people with an FH may be more susceptible to lifestyle-related risk. For example, in a pooled analysis, Cho et $a l^{8}$ reported that the relative risks of CRC with alcohol consumption of $\geq 30 \mathrm{~g} / \mathrm{d}$ were 1.23 (95\% CI 0.96 to 1.57 ; Not Significant) among those with no FH and 2.02 (95\% CI 1.30 to 3.13) among those with an FH of CRC. The importance of excess weight and increased risk of developing CRC has been highlighted by Movahedi $e t a l,{ }^{9}$ who reported that in patients with Lynch syndrome, CRC risk was increased by $7 \%$ for each $1 \mathrm{~kg} / \mathrm{m}^{2}$ increase in BMI. A recent review of alcohol, obesity and other nutritional factors in people with familial CRC by Fardet $e t a l^{10}$ present a clear case for why modifiable risk factors could offer new perspectives on prevention.

Akhtar et $a l^{1}$ reported, in a UK survey of relatives of patients diagnosed with CRC, that most (88\%) said they were prepared to make lifestyle changes if given enough information. In the BeWEL ${ }^{12}$ study (lifestyle intervention for people at higher CRC risk due to an adenoma), around half (49\%) of 997 patients showed an interest in finding out more about the lifestyle (weight loss) trial.

It is also noteworthy that, for patients subsequently diagnosed with BC or CRC, obesity is associated with poorer prognosis, increases in disease recurrence and overall mortality. ${ }^{13} 14$

Identifying increased risk of developing a disease is unlikely to be sufficient to change behaviour, ${ }^{15}$ and knowledge of FH per se is unlikely to be sufficient to initiate sustained weight management behaviours. In a questionnaire study of 237 (49\%) people attending family history clinics in the East of Scotland, Anderson et $a l^{16}$ reported that while smoking rates were modest $(11 \%)$, most respondents had a BMI $\geq 25 \mathrm{~kg} / \mathrm{m}^{2}$, low levels of physical activity and high alcohol consumption. Interview data in a subgroup of these respondents highlighted doubts about the link between lifestyle and cancer, and few were familiar with the current evidence. While lifestyle advice was considered interesting in general, there was little appetite for non-tailored guidance.

Marteau and Lerman ${ }^{17}$ argues that motivation to change behaviour may be achieved by increasing beliefs that changing behaviour can reduce risks by increasing beliefs in the individual's ability to change. However, it is more likely that self-efficacy and self-regulatory techniques (eg, goal setting) play a greater role in achieving effective behaviour change. ${ }^{18} 19$ National Institute for Health and Care Excellence guidelines on familial BC recommend that standard written information for people with concerns about familial BC risk should include 'lifestyle, including diet, alcohol etc', ${ }^{20}$ but the reasons for this choice of communication is unclear. Genetic counsellors (GCs) receive little if any training in promoting healthy lifestyles, and there is little evidence that advice is routinely provided.

This study aimed to assess the feasibility and acceptability of delivering a 12-week lifestyle intervention programme (LivingWELL) for people with an $\mathrm{FH}$ of $\mathrm{BC}$ and CRC initiated within the FH setting in order to inform the design of a definitive randomised controlled trial (RCT) to assess the clinical and cost-effectiveness of this intervention.

Specific objectives were to estimate recruitment rate for a full RCT, assess the feasibility of data collection procedures and protocol adherence, explore participant experience and establish a power calculation required for a full-scale study

\section{METHODS}

\section{Study design and setting}

This study was a two-arm, two-centre, parallel, randomised-controlled, feasibility study integrated with qualitative interviews conducted with people referred to NHS genetic services in Tayside and Grampian from August 2015 to March 2016. This study is reported in accordance with the Consolidated Standards of Reporting Trials 2010 statement: extension to randomised pilot and feasibility trials. ${ }^{21}$

\section{Sample size}

We aimed to recruit 120 (60 intervention and 60 control) participants in order to be able to assess feasibility objectives allowing for a loss to follow-up of $10 \%$ with precision of $\pm 1 \%$ at $95 \%$ CI. $^{22}$

\section{Recruitment and randomisation}

New attendees at genetics clinic were informed about the study by the GCs and given a brief information sheet, 


\begin{tabular}{|c|c|}
\hline Time line & Following baseline assessment \\
\hline Duration & $65 \mathrm{~min}$ \\
\hline Who Delivers & Trained lifestyle coach \\
\hline Location & Base (Grampian or Tayside) \\
\hline $\begin{array}{l}\text { Behavioural } \\
\text { Framework }\end{array}$ & $\begin{array}{l}\text { Leventhal's Self-Regulatory Theory } \\
\text { Social Cognitive Theory } \\
\text { Health Action Process Approach (HAPA) }\end{array}$ \\
\hline $\begin{array}{l}\text { Introduction } \\
\text { (10mins) }\end{array}$ & $\begin{array}{l}\text { Importance of lifestyle change and breast and colorectal cancer risk } \\
\text { Encouragement, Endorsement } \\
\text { Education about body weight and weight gain } \\
\text { Lifestyle and risk } \\
\text { Introduce Ten top tips as advice framework for habit development }\end{array}$ \\
\hline $\begin{array}{l}\text { Physical Activity } \\
\text { (20minutes) } \\
\text { Education and } \\
\text { interactive } \\
\text { communications } \\
\text { Behavioural } \\
\text { techniques }\end{array}$ & $\begin{array}{l}10 \text { minute walk and talk } \\
\text { Demonstration of brisk walking + pedometer } \\
\text { Benefits of exercise (beyond cancer) } \\
\text { Physical activity guidelines + Scottish Intercollegiate Guidelines Network guidelines } \\
\text { Reduce sedentary time guidance, Local facilities } \\
\text { Goal setting (using walking log) towards } 225-300 \text { minutes dependent on baseline levels. } \\
\text { Implementation intentions (make a walking plan) + coping plans }\end{array}$ \\
\hline $\begin{array}{l}\text { Diet and drinks } \\
\text { (10 minutes) } \\
\text { Education and } \\
\text { interactive } \\
\text { communications } \\
\text { Behavioural } \\
\text { techniques }\end{array}$ & $\begin{array}{l}\text { Healthy eating and weight loss principles } \\
\text { Meal routines (including breakfast) } \\
\text { Energy dense food and snacks } \\
\text { Five a day } \\
\text { Importance of water } \\
\text { Sugary and alcoholic drinks } \\
\text { Goal setting, implementation intentions only if relevant }\end{array}$ \\
\hline $\begin{array}{l}\text { Weight management } \\
\text { (20 minutes) }\end{array}$ & $\begin{array}{l}\text { 5\% weight loss prescription } \\
\text { Portion size (British Heart Foundation booklet) }\end{array}$ \\
\hline $\begin{array}{l}\text { Education and } \\
\text { interactive } \\
\text { techniques }\end{array}$ & $\begin{array}{l}\text { Identify what goals would mean to participant } \\
\text { Personalised eating plan }\end{array}$ \\
\hline $\begin{array}{l}\text { Behavioural } \\
\text { techniques }\end{array}$ & $\begin{array}{l}\text { Goal setting - realistic weight loss goal } \\
\text { Implementation intentions - self weighing weekly (log) }\end{array}$ \\
\hline $\begin{array}{l}\text { Bringing it together } \\
\text { (5minutes) }\end{array}$ & $\begin{array}{l}\text { Summarise goals } \\
\text { Check all are properly negotiated and agreed } \\
\text { Habit formation, Relapse strategies } \\
\text { All resources have been given }\end{array}$ \\
\hline Professional support & $\begin{array}{l}\text { Provide website URL and password / Provide written resources } \\
\text { Confirm telephone and email contact details } \\
\text { Explain plan for emails and calls. } \\
\text { Identify first phone call appointment } \\
\text { Introduce focus of first call and interactive task }\end{array}$ \\
\hline
\end{tabular}

Figure 1 Intervention components (face-to-face visits). BHF, British Heart Foundation.

clinician endorsement letter, non-participation questionnaire and reply slip with stamped addressed envelope. They were also offered the opportunity to discuss the study with the research team. Where low-risk patients did not attend the clinics in person, postal versions of the study materials were sent. Low initial recruitment after 3 months resulted in a protocol amendment to allow study information to be passed to established patients returning for mammographic or endoscopic procedures in one centre (not considered feasible in the other centre).

GCs/clinical staff recorded the numbers of patients attending clinics and informed patients with an FH of BC or CRC about the study. Staff noted when they felt it inappropriate to provide information about the study and recorded any reasons that patients gave for non-participation. Interested patients were given a brief information sheet and an endorsement letter from the relevant lead clinician. They were asked to provide their contact details. An opt-out slip and non-participant feedback form were included for those who decided not to participate.
Research nurses made contact with interested patients to assess eligibility and provide a full information sheet. All those written to (or given packs) could also respond by telephone/postal reply slip. Eligible participants were adults, age $\geq 18$ years, with an $\mathrm{FH}$ of BC or CRC and measured BMI $\geq 25 \mathrm{~kg} / \mathrm{m}^{2}$. Exclusion criteria were severe cognitive impairment, or conditions where physical activity was contraindicated, pregnancy, breast feeding or currently undergoing active treatment for cancer.

Once continued interest and eligibility were established, an appointment was made for a baseline measurement visit where informed consent was obtained. Postbaseline visit, participants were allocated (by the study administrator who informed the participant and lifestyle coach (LC) of the outcome) into usual care (control) or intervention groups 1:1 using a computer-generated random allocation list. The research nurse was blinded to the randomisation. 


\begin{tabular}{|c|c|}
\hline Time line & Follow on from face to face calls till 12 weeks ( 4 calls in total) \\
\hline Duration & 15 minutes \\
\hline Who delivers & Trained coach \\
\hline Call 1 (week 2) & $\begin{array}{l}\text { Check through agreed implementation intentions and progress } \\
\text { Congratulate efforts and success } \\
\text { Note down weekly weights recorded by participant } \\
\text { Identify biggest challenge - to start and to continue } \\
\text { Re-enforce modest behaviour change } \\
\text { Starting again if ill, bad weather etc.- check coping plans } \\
\text { Offer 'snack' challenge, describe briefly, provide link and discuss which snack } \\
\text { to choose. } \\
\text { Advise on how to access challenge material and agree which week to do it. } \\
\text { Make date for next call (end of week 5) where snack challenge will also be } \\
\text { discussed }\end{array}$ \\
\hline Call 2 (week 5) & $\begin{array}{l}\text { Check through agreed implementation intentions and progress } \\
\text { Congratulate efforts and success } \\
\text { Note down weekly weights recorded by participant } \\
\text { Discuss snack challenge attempts } \\
\text { Ascertain comments - what was easy and difficult } \\
\text { What would help to support the change- coping plans } \\
\text { Offer 'sitting time' challenge, describe briefly and provide link. } \\
\text { Advise on how to access challenge materials and agree which week to do it. } \\
\text { Make date for next call (end of week 9) where activity challenge will also be } \\
\text { discussed }\end{array}$ \\
\hline Call 3 (week 9) & $\begin{array}{l}\text { Check through agreed implementation intentions and progress } \\
\text { Congratulate efforts and success } \\
\text { Note down weekly weights recorded by participant } \\
\text { Discuss challenge attempts } \\
\text { Ascertain comments - what was easy and difficult } \\
\text { What would help to support the change- coping plans } \\
\text { Discuss target weight and how near they are to it } \\
\text { Identify ways in which they can maintain new habits till end of week } 12 \\
\text { Make date for next call (end of week 12) }\end{array}$ \\
\hline Call 4 (week 12) & $\begin{array}{l}\text { Farewell and thank you call } \\
\text { Discuss long term planning -weight over next year } \\
\text { How could we support long term weight goals } \\
\text { Is there further support we can provide through the web }\end{array}$ \\
\hline
\end{tabular}

Figure 2 Intervention components (telephone calls).

\section{Intervention}

The 12-week intervention, 'LivingWELL', aimed to help participants increase physical activity, modify diet, achieve weight loss towards $5 \%$ of body weight and avoid weight gain. Personalised advice was given on a $600 \mathrm{kcal}$ deficit dietary intake as recommended by the Scottish Intercollegiate Guidelines Network (SIGN) ${ }^{23}$ and a graduated approach aimed at increasing activity to 225-300 min per week by 12 weeks. The theoretical basis for the intervention draws on Leventhal's Self-Regulatory Theory ${ }^{24}$ (which highlights the importance of the individual's beliefs regarding illness cause, identity, control/cure, timeline and consequences), Social Cognitive Theory, ${ }^{25}$ which emphasises the importance of self-efficacy, and the Health Action Process Approach, ${ }^{26}$ which emphasises the importance of action and coping planning.

The 'LivingWELL' programme was delivered by LCs personnel (with a nursing background) who received bespoke training on the delivery of the intervention programme. Intervention participants received one face-to-face session plus (up to) four telephone consultations over the 12-week programme, a 'LivingWELL' information pack, pedometer and walking programme. Participants were also offered a web-based support programme. The face-to-face session was designed to be interactive and included a $10 \mathrm{~min}$ 'walk and talk' session during which pedometer use and walking goals were discussed, self-identification of BMI category and a portion weight estimate task.

Participants received a personalised energy deficit diet, a personalised graduated walking plan and guidance on setting personal goals, how to make changes habitual and relapse prevention strategies for times of deviation.

Motivational interviewing techniques were used to explore self-assessed confidence to change and self-perceived benefits. Behavioural change techniques (goal setting, action and coping plans and implementation intentions) were also used. The importance of self-monitoring pedometer data, diet and drink logs and weekly body weight was emphasised. These data formed the 
Table 1 Demographic characteristics at baseline

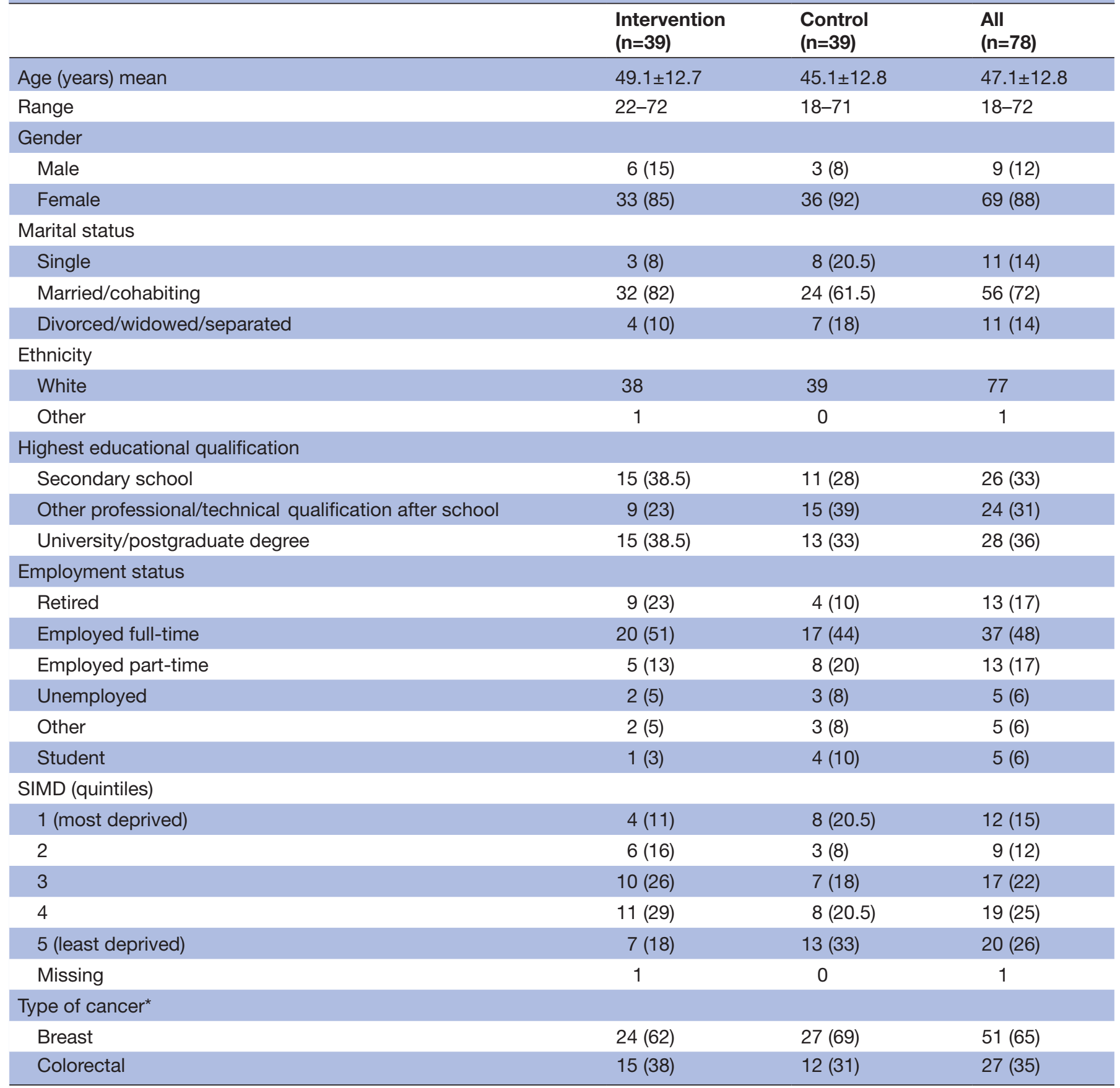

${ }^{*}$ Cancer family history, which resulted in a genetics referral.

All results are $n(\%)$ unless stated otherwise.

SIMD, Scottish Index of Multiple Deprivation.

basis for the 15 min phone consultations, which checked well-being, self-monitoring behaviours, actions and coping plans. Details of the intervention are described in figures 1 and 2.

\section{Primary outcome measure}

Data were collected on numbers of people told about the study, those expressing an interest, numbers meeting eligibility criterion, rates of recruitment, achieved measurements and retention. Reasons for non-participation and withdrawal were collected where possible.

Intervention contact time was recorded by LCs, and the ease of implementing the counselling sessions and perceived engagement and motivation of participants was recorded using a 5-point Likert scale (scored -2 to +2 ). A random sample of face-to-face visits and telephone calls were recorded and independently analysed to assess fidelity to the detailed intervention protocol. 


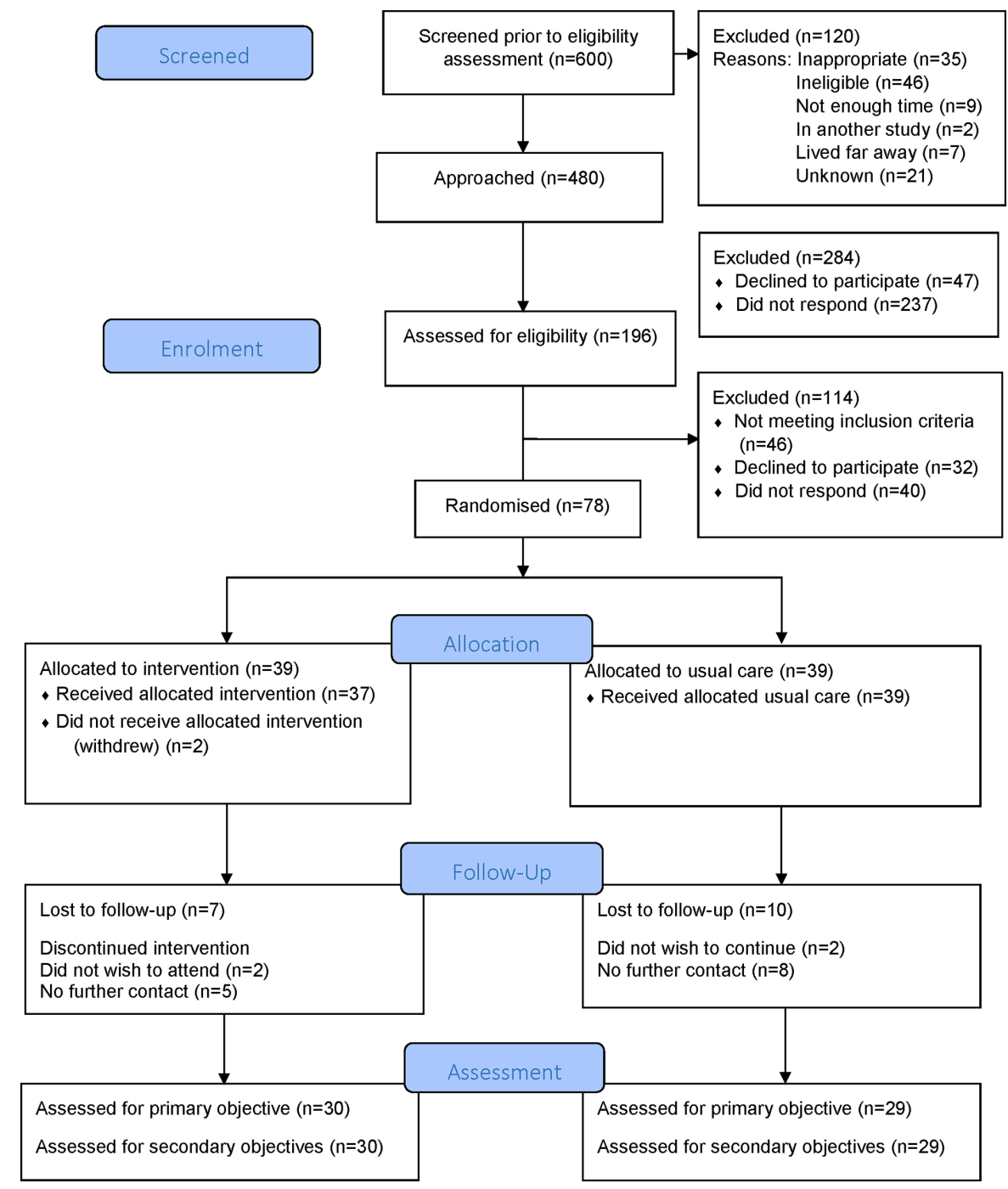

Figure 3 CONSORT diagram 'LivingWELL' total recruitment. CONSORT, Consolidated Standards of Reporting Trials.

All participants who completed the study were asked to fill out an anonymous patient satisfaction questionnaire (to rate programme visits, measurements and intervention) that was distributed after the follow-up visit. Qualitative interviews $(\mathrm{n}=20)$ explored intervention participants' views on study acceptability (recruitment, assessment and intervention programme) and factors influencing adherence. Interviews were audio recorded, transcribed and analysed using a thematic framework approach.

\section{Secondary outcome measures}

Follow-up procedures took place after the 12-week intervention period. Both baseline and follow-up measures were undertaken by research nurses blinded to the group allocation, and participants were asked not to share their group allocation with the researchers.

Research nurses recorded sociodemographic information and measured height at the baseline visit. At both baseline and follow-up, physiological measures (body weight, waist circumference and blood pressure) were taken, and BMI was assessed.
Physical activity was subjectively assessed using the International Physical Activity Questionnaire Short Form ${ }^{27}$ (for low/medium and high activity grading and to get insight into activity modes) and objectively measured by Sensewear (BodyMedia, Pittsburgh, Pennsylvania, USA) physical activity monitors, which provided detailed analysis of sedentary time, moderate and vigorous activity and step counts. Eating habits were assessed by the Dietary Instrument for Nutrition Education questionnaire ${ }^{28}$ and alcohol intake by a 7-day alcohol record. ${ }^{29}$ Smoking was assessed by questions on current and historic tobacco use.

Psychosocial variables measured included beliefs about cancer cause and risk reduction (modified brief illness perception questionnaire ${ }^{30}$ ) and quality of life using the Euro-Qol-5D questionnaire. ${ }^{31}$ These measures were undertaken primarily to assess participant burden for planning a full-scale trial.

\section{Statistical analysis}

For all quantitative outcome measures, the main aim of this study was principally to assess feasibility of intervention 
Table 2 Changes in physiological measures

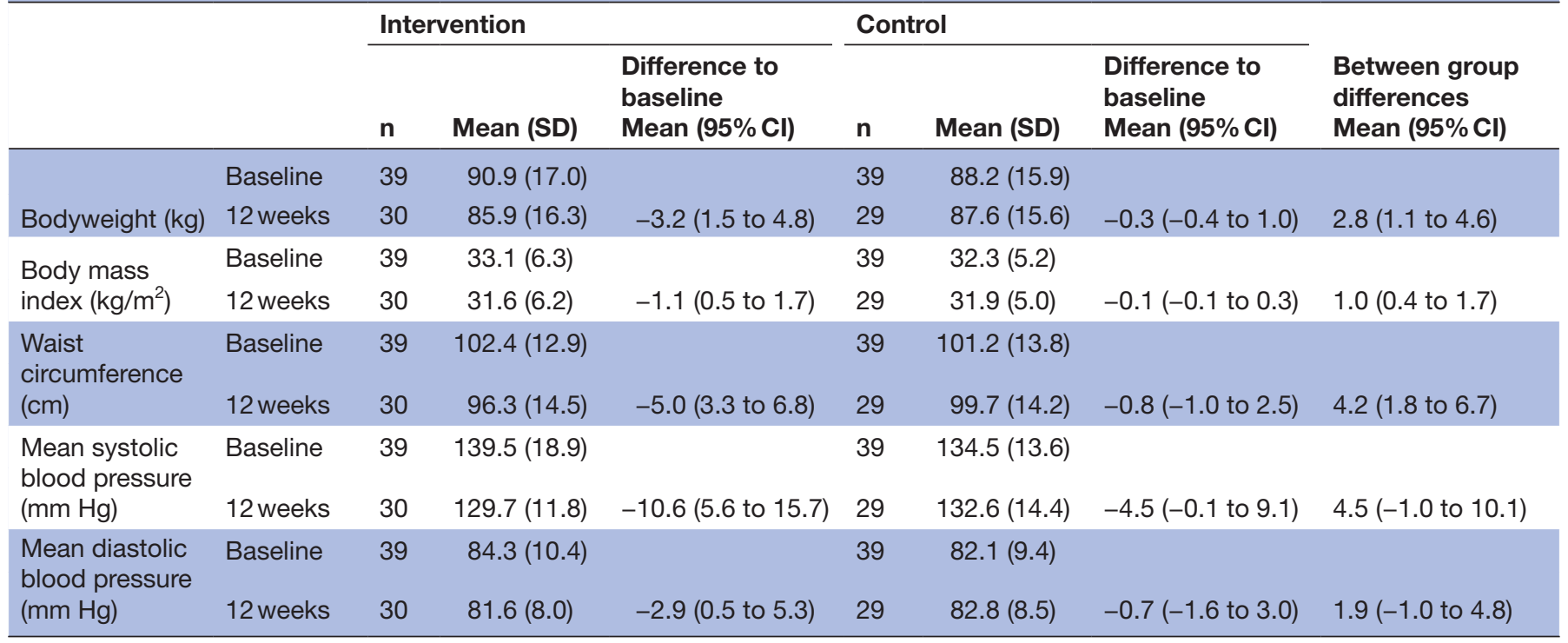

delivery to inform the design of a main trial, thus the indicative outcomes are underpowered for statistical interpretation.

Quantitative data analysis was undertaken using STATA V.14. Descriptive statistics were used to characterise the cohort. Within group changes between baseline and follow-up outcomes were estimated for intervention and control groups using a paired t-test. Between group differences in the outcome change from baseline and follow-up adjusted for baseline values were estimated using linear regression methods. Both within and between group differences are presented as means and 95\% CIs.

\section{RESULTS}

Primary outcomes (feasibility)

Patients informed about the study

In total, 600 patients were identified as potentially eligible for the study (364 BC and 236 CRC FH) over the 8-month recruitment period (figure 3). Of those, 312 (52\%) were new patients and $288(48 \%)$ review patients (4month period only). Records kept by clinical and study staff show a total of 480 patients (282 BC and $198 \mathrm{CRC}$ FH) were given study information (169 in person and 311 by invitation letters). Within the clinics, 120 people were not approached where GCs considered it inappropriate (35), the person was thought to be ineligible (46), there was not enough time (9), patients were in another study (2), staff noted they lived far from the hospital (7) or for reasons unknown (21).

\section{Patients interested in the study}

A total of $196(41 \%)$ people (132 BC and 64 CRC FH) agreed to be contacted. Greatest interest in the study was expressed by new and review patients advised about the study in face-to-face clinic visits $(70 \%$ and $93 \%$, respectively, agreed to be contacted) compared with new and review patient advised by letter $(7 \%$ and $27 \%$, respectively). It is notable that despite the high interest expressed by review patients in face-to-face visits (93\%), the numbers randomised dropped to $32 \%$. No new patients contacted by letter went on to be randomised.

\section{Eligibility and recruitment rate}

Of the 196 who agreed to be contacted, $32(16 \%)$ declined to take part, 40 (20\%) did not respond to telephone approaches and 46 (23\%) were ineligible (due to $\mathrm{BMI}$ ). A total of 78 people ( $40 \%$ of those who agreed to be contacted; $39 \%$ of BC and $42 \%$ of CRC FH approached; $16 \%$ of those who were potentially eligible for the study) were randomised.

\section{Baseline characteristics}

The mean age was $27.1+12.8$ years (range 18-72) and $88 \%$ were female (table 1). Most were in employment, had postschool professional or academic qualifications and belonged to the two least deprived quintiles of the Scottish Index of Multiple Deprivation (SIMD).

\section{Achieved measurements}

Evaluation measures were $99 \%-100 \%$ complete for all participants who attended baseline and follow-up appointments. Accelerometer data (for at least 4 days) was obtained for 67 (84\%) participants at baseline and 42 (54\%) at follow-up.

\section{Retention}

Of the 78 randomised, $59(75 \%)$ completed the study (74\% control and $77 \%$ intervention). Completion rates were similar by BC (72\%) and CRC (81\%) (figure 3). A greater proportion of people from SIMD quintiles 1 and 2 (high deprivation) were likely to withdraw compared with SIMD quintiles 3, 4 and 5 (38\% vs 22\%).

\section{Non-participation}

It is estimated that approximately 100 non-participation feedback forms were distributed at clinic appointments, and over 300 were posted with study information packs. A 
Table 3 Changes in physical activity

\begin{tabular}{|c|c|c|c|c|c|c|c|c|}
\hline & & \multicolumn{3}{|c|}{ Intervention } & \multicolumn{3}{|c|}{ Control } & \multirow[b]{2}{*}{$\begin{array}{l}\text { Between group } \\
\text { differences } \\
\text { Mean }(95 \% \mathrm{Cl})\end{array}$} \\
\hline & & $\mathbf{n}$ & Mean (SD) & $\begin{array}{l}\text { Difference to } \\
\text { baseline } \\
\text { Mean }(95 \% \mathrm{Cl})\end{array}$ & $\mathbf{n}$ & Mean (SD) & $\begin{array}{l}\text { Difference to } \\
\text { baseline } \\
\text { Mean }(95 \% \mathrm{Cl})\end{array}$ & \\
\hline $\begin{array}{l}\text { Daily average } \\
\text { time spent in } \\
\text { moderate activity } \\
\text { (min) }\end{array}$ & 12 weeks & 23 & $86.8(62.0)$ & $20.3(-36.2$ to -4.4$)$ & 38 & $60.3(39.4)$ & $5.82(-27.2$ to 15.5$)$ & -13.6 (39.0 to 11.7$)$ \\
\hline \multirow{2}{*}{$\begin{array}{l}\text { Daily average } \\
\text { step count }\end{array}$} & Baseline & 34 & $7544(3738)$ & & 38 & 7999 (3937) & & \\
\hline & 12 weeks & 23 & $10315(5551)$ & $1760(-3111$ to -408$)$ & 23 & 9468 (3793) & 699 (-2629 to 1230$)$ & 939 (-3187 to 1309$)$ \\
\hline
\end{tabular}

total of 28 questionnaires were returned. Most responders were women $(86 \%)$, and the average age was 51.2 years (range 25-72). Of those, 21 people reported a weight and height: 9 had a normal BMI and 12 were overweight or obese. The majority of respondents were from areas of low deprivation (59\%). The most common reasons for not taking part were that 'their lifestyle was already good' $(29 \%)$ and 'too little time to participate' (14\%).

\section{Intervention contact and delivery}

In the intervention group, 37 of the 39 randomised (two withdrew) received a face-to-face session with a LC. The mean time for the face-to-face visit was $81 \mathrm{~min}$ (range 70-130) compared with an estimated $65 \mathrm{~min}$. The mean time for telephone calls at all time points was $15 \mathrm{~min}$ (range 5-30), the same as the estimated allowance.

The LCs estimated around $73 \%$ of the total programme was covered, while fidelity scores for nine face-to-face sessions and that 16 telephone calls rated that $62 \%$ of the intervention components were delivered as per protocol. LCs assessed perceived patient engagement, receptivity and motivation highly: $89 \%, 92 \%$, and $89 \%$ respectively, in Likert-scaled questions.

Access to the 'LivingWELL' study website was given to all intervention participants and $10(33 \%)$ logged on.

\section{Participant feedback}

Acceptability data were obtained from 47 (80\%) anonymised patient satisfaction questionnaires (23 control and 24 intervention) and from the 20 participant interviews. Results from the questionnaire showed that most (89\%) participants reported that they found study participation quite/very easy and $38(81 \%)$ reported that they would recommend 'LivingWELL' to others. Most $(80 \%)$ of the intervention group expressed a high degree of satisfaction with intervention components. The following suggestions for change were made: feedback of results, further contacts with coaches and longer term follow-up.

The qualitative interview data revealed that intervention staff were perceived by participants as professional, knowledgeable, helpful and approachable. Appointments at the centre with LCs were largely perceived as convenient in terms of duration, scheduling and travel incurred. The various measurements were not considered invasive or inappropriate. However, a few participants found the activity monitors heavy and uncomfortable. Overall, most participants were satisfied with the study and recommended few improvements. Participants reported the following barriers to change:

- everyday routines

- sedentary occupations

- family commitments

- poor physical or mental health

- stressful events such as exams, bereavement or family illness

- long-standing complex relationships with food.

In general, participants appeared to find it easier to change physical activity than diet.

\section{Secondary outcomes}

The aim of this study was to determine feasibility, not to evaluate statistically significant change. However, changes in physiological measures suggest a favourable effect of the intervention on the primary outcome of body weight loss and diastolic blood pressure (table 2). These findings are reflected in the proportions achieving $5 \%$ weight loss $(37 \%$ in the intervention group compared with $0 \%$ in control group) and $10 \%$ weight loss (10\% of the intervention group).

Favourable increases in moderate physical activity (from objective accelerometer data) and decreases in dietary fat scores were also reported (tables 3 and 4). Little change was observed in reported alcohol intake.

Using the data from the current study, where we observed a mean body weight of $89.5 \mathrm{~kg}( \pm$ SD 13.3), a total of 187 participants per group would be needed to detect a between group difference of $5 \%$ weightt change $(4.47 \mathrm{~kg})$ at follow-up, at $90 \%$ power and $5 \%$ alpha based on a two-tailed unpaired t-test. Allowing for an assumed $25 \%$ drop-out this would mean recruiting 250 participants per group.

Based on the current figures: to recruit 500 people, 1250 would need to express an interest $(40 \%$ of those 
Table 4 Changes in dietary intake scores

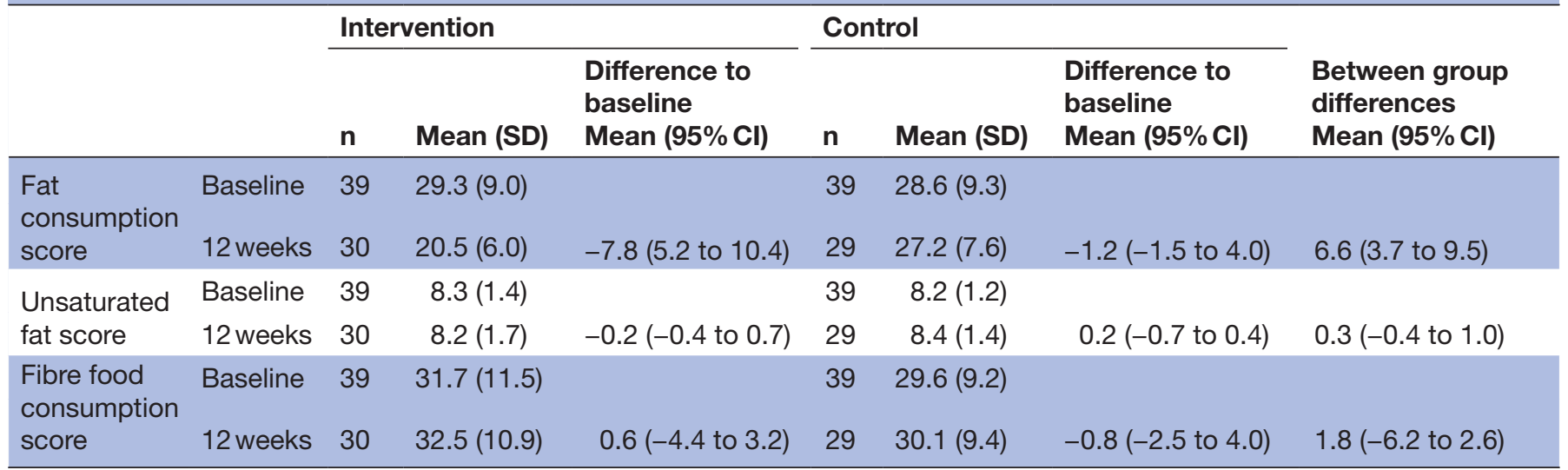

who expressed an interest were recruited). For 1250 to express an interest, 3048 would require to be approached (based on $41 \%$ of people approached were interested). If all clinics recruited similar numbers to the two in the current study (240 in an 8-month period/360 per year), then nine centres would be needed for a 12-month recruitment period.

\section{DISCUSSION}

\section{Main findings}

Despite evidence for the importance of lifestyle in the aetiology of BC and CRC, counselling on diet, physical activity and weight management is not routinely provided for people with a family history of these conditions referred to NHS genetics clinics. While cancer preventability estimates suggest that healthful ways of life could significantly reduce cancer risk, the impact of lifestyle interventions in this patient group is unknown and randomised controlled trial data is needed to examine the cost, benefits and harms. The results from this pilot study show that it is feasible to recruit, randomise and retain patients and to implement and evaluate a weight management programme combining diet and physical activity advice with behavioural change techniques that is acceptable to patients. The results also indicate that implementation of this programme is associated with favourable outcomes. However, the current work has highlighted the importance of face-to-face approaches for study recruitment and the cautious approach taken by genetic clinic staff to introducing the study. The findings have also enabled the collection of data that allows a power calculation to be performed for the design of a definitive randomised controlled trial.

\section{Strengths and weaknesses}

The main strength of the study is that (to the best of our knowledge) the current work is the first attempt to offer a comprehensive lifestyle programme (diet, alcohol, physical activity and body weight) for people referred to $\mathrm{FH}$ clinics for $\mathrm{CRC}$ and $\mathrm{BC}$ risk assessment. The main weakness of the study is the sociodemographic profile of participants recruited who were predominantly Caucasian and $>50 \%$ were from the two lowest deprivation quintiles (eg, higher socioeconomic status). Although this distribution reflects the general demographic for high-risk attendees for breast mammography clinics in this region $^{32}$ (where there is little ethnic diversity), it highlights the difficulties in offering both surveillance and lifestyle interventions to affected people from more deprived areas.

The sample size was less than planned due to fewer patients being referred to the service than previous years (on which our estimate was based), fewer informed about the study than estimated (despite input from clinic staff in study design) and fewer being seen face to face than anticipated. The current results underline the need for feasibility work. Overall, study retention was high but further efforts to reduce loss to follow-up in people living in areas of higher deprivation should be explored. Attempts to increase recruitment from existing patients demonstrate that both new and returning patients have some interest in the topic, but in general a greater awareness of the relevance of lifestyle in cancer prevention would be beneficial to recruitment. Other weaknesses include the time allocation for the face-to-face meeting, which was insufficient for the intended content, resulting in the LCs deviating from the original protocol. In addition, retention was only assessed over a 12-week period, and a longer test period is likely to be associated with higher loss to follow-up. Independent assessment of fidelity also suggests scope for improvement in time allocation as only $62 \%$ of intervention components were delivered per protocol.

\section{Other studies}

No fully powered trials of lifestyle interventions in people with family history of cancer have yet reported and those that have been described in the literature focus primarily on physical activity ${ }^{34}$ in Breast Cancer, early onset (BRCA) mutation carriers. Ongoing trials of weight management to decrease $\mathrm{BC}$ risk are being undertaken in women already diagnosed with the disease ${ }^{35-38}$ but are not exclusive to people at increased genetic risk. Pilot and 
formative work on weight loss and reducing markers of BC has been undertaken ${ }^{39}$ (notably on the intermittent energy diet approach) in women with family history of BC, demonstrating significant changes in insulin sensitivity and body fat reduction, but no work has been reported for patients with a family history of CRC. Postintervention interviews with participants ${ }^{40}$ have highlighted the importance of providing a credible rationale for weight control and weight loss that underlines the need for health professionals working in this area to introduce and endorse the importance of lifestyle to aid recruitment.

\section{Implications for clinicians and policymakers}

This pilot study has highlighted a number of perceived challenges for NHS staff discussing lifestyle issues among patients with a family history of BC and CRC but has also demonstrated that there is an interest in being able to offer more help that could usefully be developed. Indicative results suggest the intervention had a favourable effect on body weight, physical activity and fat intake score with potential for clinically relevant benefits for both cancer risk reduction and long-term health (with or without a cancer diagnosis). While the qualitative data highlight the complexity and challenge of changing health behaviours, the measured changes in physical activity and weight loss suggest that small but significant changes can be achieved, at least in the short term. Support for habitual and sustained lifestyle change is essential as part of longterm intervention design.

\section{Unanswered questions and future research}

The generalisability of the current findings are unclear. However, work to date suggests that the intervention is feasible to deliver and evaluate and could be tested in a large RCT, subject to modifications. In a fully powered trial, the first stage would be to assess the magnitude of lifestyle change that can be achieved by this type of programme. In turn, this fully powered trial would act as a 'pilot' for a full trial of reduction in CRC markers. Adenomas (number and size) might be an appropriate end point depending on funding for the length of follow-up.

It would be desirable to increase patient recruitment, and the current findings suggest that overall uptake could be increased with better training, support and endorsement from the GCs and other clinical staff. This area of study was almost entirely new and met with scepticism from staff and indeed patients. Our earlier work suggests ambiguous attitudes about the importance of lifestyle with little evidence that these topics have been previously discussed with clinicians. ${ }^{18}$

The programme was received enthusiastically by participants, but further work is needed to refine intervention components, particularly dietary aspects that were less appreciated than the physical activity aspects and may need tailoring for ethnic groups. Measurements were successfully attained and appropriate for a full study protocol, although a review of accelerometry approaches is warranted. The numbers required for a full trial with weight change as a primary outcome would require a multicentre approach with a minimum recruitment period of at least 12 months. Overall, the main uncertainties required for planning a definitive randomised controlled trial have been addressed.

\section{Author affiliations}

${ }^{1}$ Centre for Research into Cancer Prevention and Screening / Tayside Cancer Centre, Division of Cancer Research, University of Dundee, Ninewells Hospital \& Medical School, Dundee, UK

${ }^{2}$ Department of Clinical Genetics, Ninewells Hospital and Medical School, Dundee, UK

${ }^{3}$ Clinical Genetics Service, Ashgrove House, Foresterhill, Aberdeen, UK

${ }^{4}$ Physical Activity for Health Research Centre, Institute for Sport, Physical Education and Health Sciences, University of Edinburgh, Edinburgh, UK

${ }^{5}$ Division of Psychology, School of Natural Sciences, University of Stirling, Stirling, UK

${ }^{6}$ Institute for Social Marketing, Institute for Social Marketing, University of Stirling, Stirling, UK

${ }^{7}$ Institute of Health Research, University of Exeter Medical School, Exeter, UK

${ }^{8}$ Department of Genetic Medicine, Ninewells Hospital and Medical School, Dundee, UK

Acknowledgements The authors would like to acknowledge the valuable contribution given by Heather Gow (research nurse), Maria Nika and Catherine Savage (lifestyle coaches) and all centre staff involved in the study.

Contributors ASA and RJCS had the original idea for the study and, with JD, MM, ZM, NM, REO, MS, SV and JB, designed the trial parameters and formed the investigator group who obtained the funding. ASA, JB, JD, ZM, REO, SG and MS were responsible for overseeing study implementation and data collection. MM, REO and RST carried out the analysis. ASA and MM drafted the manuscript, which was revised by all authors. All authors approved the final paper and were independent from funders.

Funding This work was supported by Chief Scientist Office for Scotland, grant number $\mathrm{CZH} / 4 / 1080$.

Competing interests None declared.

Patient consent Obtained.

Ethics approval Ethical approval for this study was provided by East of Scotland Research Ethics Service (REC reference: 15/ES/0055).

Provenance and peer review Not commissioned; externally peer reviewed.

Data sharing statement Technical appendix, statistical code and dataset available from the authors on request.

Open Access This is an Open Access article distributed in accordance with the Creative Commons Attribution Non Commercial (CC BY-NC 4.0) license, which permits others to distribute, remix, adapt, build upon this work non-commercially, and license their derivative works on different terms, provided the original work is properly cited and the use is non-commercial. See: http://creativecommons.org/ licenses/by-nc/4.0/

(c) Article author(s) (or their employer(s) unless otherwise stated in the text of the article) 2018. All rights reserved. No commercial use is permitted unless otherwise expressly granted.

\section{REFERENCES}

1. Genetic Alliance UK [Internet]. NHS Genetics services in the UK. 2017 http://www.geneticalliance.org.uk/information/services-andtesting/nhs-genetic-services-in-the-uk/ (cited Aug 2017).

2. World Cancer Research Fund [Internet]. Cancer Preventability Statistics. https://www.wcrf-uk.org/uk/preventing-cancer/cancerpreventability-statistics (cited 2017 Aug 7).

3. Harvie M, Howell A, Vierkant RA, et al. Association of gain and loss of weight before and after menopause with risk of postmenopausal breast cancer in the lowa women's health study. Cancer Epidemiol Biomarkers Prev 2005;14:656-61. 
4. Ahn J, Schatzkin A, Lacey JV, et al. Adiposity, adult weight change, and postmenopausal breast cancer risk. Arch Intern Med 2007;167:2091-102.

5. Schauer DP, Feigelson HS, Koebnick C, et al. Bariatric surgery and the risk of cancer in a large multisite cohort. Ann Surg 2017. [Epub ahead of print 21 Sep 2017].

6. Gramling R, Lash TL, Rothman KJ, et al. Family history of later-onset breast cancer, breast healthy behavior and invasive breast cancer among postmenopausal women: a cohort study. Breast Cancer Res 2010;12:R82.

7. Pettapiece-Phillips R, Narod SA, Kotsopoulos J. The role of body size and physical activity on the risk of breast cancer in BRCA mutation carriers. Cancer Causes Control 2015;26:333-44.

8. Cho E, Lee JE, Rimm EB, et al. Alcohol consumption and the risk of colon cancer by family history of colorectal cancer. Am J Clin Nutr 2012;95:413-9.

9. Movahedi M, Bishop DT, Macrae F, et al. Obesity, aspirin, and risk of colorectal cancer in carriers of hereditary colorectal cancer: a prospective investigation in the CAPP2 study. $\mathrm{J}$ Clin Oncol 2015;33:3591-7.

10. Fardet A, Druesne-Pecollo N, Touvier M, et al. Do alcoholic beverages, obesity and other nutritional factors modify the risk of familial colorectal cancer? A systematic review. Crit Rev Oncol Hematol 2017;119:94-112.

11. Akhtar S, Sinha S, McKenzie S, et al. Awareness of risk factors amongst first degree relative patients with colorectal cancer. Colorectal Dis 2008;10:887-90.

12. Anderson AS, Craigie AM, Caswell S, et al. The impact of a bodyweight and physical activity intervention (BeWEL) initiated through a national colorectal cancer screening programme: randomised controlled trial. BMJ 2014;348:g1823.

13. Meyerhardt JA, Catalano PJ, Haller DG, et al. Influence of body mass index on outcomes and treatment-related toxicity in patients with colon carcinoma. Cancer 2003;98:484-95

14. Chan DS, Vieira AR, Aune D, et al. Body mass index and survival in women with breast cancer-systematic literature review and metaanalysis of 82 follow-up studies. Ann Oncol 2014;25:1901-14.

15. French DP, Cameron E, Benton JS, et al. Can communicating personalised disease risk promote healthy behaviour change? A systematic review of systematic reviews. Ann Behav Med 2017;51:718-29.

16. Anderson AS, Caswell S, Macleod M, et al. Health behaviors and their relationship with disease control in people attending genetic clinics with a family history of breast or colorectal cancer. $J$ Genet Couns 2017;26:40-51.

17. Marteau TM, Lerman C. Genetic risk and behavioural change. BMJ 2001:322:1056-9.

18. Dombrowski SU, Sniehotta FF, Avenell A, et al. Identifying active ingredients in complex behavioural interventions for obese adults with obesity-related co-morbidities or additional risk factors for comorbidities: a systematic review. Health Psychol Rev 2012;6:7-32.

19. Michie S, Richardson M, Johnston M, et al. The behavior change technique taxonomy (v1) of 93 hierarchically clustered techniques: building an international consensus for the reporting of behavior change interventions. Ann Behav Med 2013;46:81-95.

20. National Institute for Clinical and Health Excellence. Familial breast cancer. NICE clinical guideline 164. 2017 https://www.nice.org.uk/ guidance/cg164/chapter/Recommendations\#clinical-significance-ofa-family-history-of-breast-cancer.
21. Eldridge SM, Chan CL, Campbell MJ, et al. CONSORT 2010 statement: extension to randomised pilot and feasibility trials. Pilot Feasibility Stud 2016;2:64.

22. Sim J, Lewis M. The size of a pilot study for a clinical trial should be calculated in relation to considerations of precision and efficiency. $J$ Clin Epidemiol 2012;65:301-8.

23. Scottish Intercollegiate Guideline Network (SIGN). Management of Obesity. Edinburgh: SIGN, 2010.

24. Leventhal H, Leventhal EA, Breland JY. Cognitive science speaks to the "common-sense" of chronic illness management. Ann Behav Med 2011;41:152-63.

25. Bandura A. Social Foundations of Thought and Action: A social cognitive theory. Englewood Cliffs, NJ: Prentice-Hall, 1986.

26. Schwarzer R. Modeling health behaviour change: how to predict and modify the adoption and maintenance of health behaviors. Appl Psychol 2008;57:1-29.

27. Craig CL, Marshall AL, Sjöström M, et al. International physical activity questionnaire: 12-country reliability and validity. Med Sci Sports Exerc 2003;35:1381-95.

28. Roe L, Strong C, Whiteside $\mathrm{C}$, et al. Dietary intervention in primary care: validity of the DINE method for diet assessment. Fam Pract 1994;11:375-81.

29. Emslie C, Lewars H, Batty GD, et al. Are there gender differences in levels of heavy, binge and problem drinking? Evidence from three generations in the west of Scotland. Public Health 2009;123:12-14.

30. Broadbent E, Petrie KJ, Main J, et al. The brief illness perception questionnaire. J Psychosom Res 2006;60:631-7.

31. EQ-5D 2017. Euroqol organisation [Internet]. https://euroqol.org/ (cited Aug 2017)

32. NHS Tayside. The Business Unit Information Team. Heraklion, Greece: The European Union Agency for Network and Information Security, 2014.

33. Pasanisi P, Bruno E, Manoukian S, et al. A randomized controlled trial of diet and physical activity in BRCA mutation carriers. Fam Cancer 2014;13:181-7

34. Kiechle M, Engel C, Berling A, et al. Lifestyle intervention in BRCA1/2 mutation carriers: study protocol for a prospective, randomized, controlled clinical feasibility trial (LIBRE-1 study). Pilot Feasibility Stud 2016;2:74.

35. Pegington M, Adams JE, Bundred NJ, et al. Recruitment to the "Breast-Activity and Healthy Eating After Diagnosis" (B-AHEAD) Randomized Controlled Trial. Integr Cancer Ther 2017;1:153473541668785.

36. Clinical Trials.Gov [Internet]. Breast Cancer WEight Loss Study (BWEL Study). https://clinicaltrials.gov/ct2/show/NCT02750826 (Cited 7 Aug 2017).

37. Clinical Trials.Gov [Internet]. Weight Gain Prevention for Breast Cancer Survivors. https://clinicaltrials.gov/ct2/show/NCT00533338 (Cited Aug 2017).

38. Gnagnarella $P$, Dragà $D$, Baggi $F$, et al. Promoting weight loss through diet and exercise in overweight or obese breast cancer survivors (InForma): study protocol for a randomized controlled trial. Trials 2016;17:363.

39. Harvie $M$, Wright $C$, Pegington $M$, et al. The effect of intermittent energy and carbohydrate restriction $\mathrm{v}$. daily energy restriction on weight loss and metabolic disease risk markers in overweight women. Br J Nutr 2013;110:1534-47.

40. Wright CE, Harvie M, Howell A, et al. Beliefs about weight and breast cancer: an interview study with high risk women following a 12 month weight loss intervention. Hered Cancer Clin Pract 2015;13:1. 